\title{
Mathematical Model Analysis and Simulation of Visceral Leishmaniasis, Kashgar, Xinjiang, 2004-2016
}

\author{
Yateng Song, ${ }^{1}$ Tailei Zhang, ${ }^{2}$ Hui Li $\left(\mathbb{D},{ }^{3}\right.$ Kai Wang $\mathbb{D},{ }^{4}$ and Xiaobo Lu ${ }^{5}{ }^{5}$ \\ ${ }^{1}$ College of Public Health, Xinjiang Medical University, Urumqi 830011, China \\ ${ }^{2}$ School of Science, Chang'an University, Xi'an 710064, China \\ ${ }^{3}$ Central Laboratory, Xinjiang Medical University, Urumqi 830011, China \\ ${ }^{4}$ College of Medical Engineering and Technology, Xinjiang Medical University, Urumqi 830011, China \\ ${ }^{5}$ Department of Infectious Diseases, The First Affiliated Hospital of Xinjiang Medical University, Urumqi 830054, China \\ Correspondence should be addressed to Kai Wang; wangkaimath@sina.com and Xiaobo Lu; xjykdluxiaobo@126.com
}

Received 12 September 2019; Revised 3 January 2020; Accepted 11 February 2020; Published 24 April 2020

Guest Editor: George V. Popescu

Copyright (C) 2020 Yateng Song et al. This is an open access article distributed under the Creative Commons Attribution License, which permits unrestricted use, distribution, and reproduction in any medium, provided the original work is properly cited.

\begin{abstract}
Visceral leishmaniasis (VL), known as kala-azar, is a serious parasitic disease. After malaria, VL is the second largest parasitic killer. This paper focuses on the VL transmission around sandflies, dogs, and people. Kashgar is located on the southwestern edge of Xinjiang, where kala-azar parasite infection occurs every year. According to the cases reported in the Kashgar Prefecture from 2004 to 2016, we proposed a dynamic model based on these three populations. The SEIR model was established for human population, the SI model was established for sandfly population, and the SI model was established for dog population. We fitted the model to cumulative cases from 2004 to 2016 for the epidemic in Kashgar and predicted that the cumulative incidence of kalaazar in Kashgar would continue to increase, but its growth rate would gradually slow down, which means that the number of cases would gradually decrease every year. We also estimated the basic reproduction number $R_{0}=1.76$ (95\% CI: 1.49-1.93). The sensitivity analysis shows that the mutual infection between sandfly and dog contributes the most to the basic reproduction number, while the transmission proportion of sandfly to the susceptible person and the mutual infection between sandfly and dog contribute the most to the number of leishmaniasis human cases. Therefore, according to the sensitivity analysis results, reducing the contact between sandflies and dogs is an effective way to reduce kala-azar.
\end{abstract}

\section{Introduction}

Visceral leishmaniasis (VL) is also called kala-azar, which is a chronic infectious disease caused by Leishmania infantum. VL has ranked as the second largest parasitic killer after malaria and draws worldwide attention because of its severity; about 12 million people are affected by it around the world [1]. The pathogen causing visceral leishmaniasis are $L$. donovani, L. infantum, and L. chagasi. The incubation period for VL is generally $3-6$ months, at least 10 days, while the longest is 9 years [1]. Humans and dogs are the main infection sources while sandflies are the main carriers.

The World Health Organization (WHO) lists leishmaniasis as the most easily neglected disease. It is reported that approximately $30 \%$ of new clinical cases and 58,000 deaths of leishmaniasis occur worldwide each year [2]. According to the characteristics of the source of infection, VL is mainly divided into three types: human-borne, canine-borne, and wildlifeborne in China [3]. In the early days of the People's Republic of China, human-borne kala-azar was the main disease in 16 provinces, such as Shandong and Henan. After prevention and treatment, it was basically eliminated. In recent years, VL has been prevalent in six provinces including Xinjiang, Inner Mongolia, Gansu, Sichuan, Shaanxi, and Shanxi [3]. Among six provinces, the typical VL in Xinjiang is wildlife-borne, and some areas have human-borne VL, while canine-borne is the main transmission way in Gansu.

Xinjiang is the largest provincial administrative region in China and is also a high-risk area for VL, especially in the Kashgar Prefecture in southern Xinjiang. The Kashgar Prefecture is located in the southwestern margin of the Xinjiang Uygur Autonomous Region. Due to its unique geographical 
environment, there are two types of visceral leishmaniasis, human and desert [4]. Since the 1990s, the incidence of VL in Kashgar has been increasing year by year. From 2005 to 2015, Xinjiang's VL has experienced significant fluctuation. In 2007-2012, the incidence of VL in Kashgar accounted for more than $90 \%$ of the total population [5].

We utilized the data of human leishmaniasis cases from 2004 to 2016 reported by the Center for Disease Control and Prevention in Xinjiang, and then we plot bar diagrams about total, sex, and age which are presented in Figures 1(a)-1(c). From Figure 1(a), we could find that the reported cases of VL in Kashgar Prefecture of Xinjiang fluctuate considerably, with outbreaks occurring in October and November of 2008, 2009, and 2015, respectively. From Figure 1(b), it shows that there are more reported cases of VL in males than in females. Figure 1(c) shows that there are more reported cases of VL in the younger age group.

In recent years, a lot of mathematical models have been established around humans, dogs, and sandflies to understand the transmission dynamics of VL (see for instance [6-14]). For example, Muhammad and Ali [6] considered the uniform mixing of the population, established the SEIHR model for the population and the SEIR model for the dog, which used sandfly as the medium, modeled and backwarded the branching of zoonotic visceral leishmaniasis, and calculated the basic reproduction number $R_{0}$ for optimal control. Elmojtaba et al. [7] developed a mathematical model to study the dynamics of visceral leishmaniasis in Sudan, considering three different groups, analyzing the balance point and its stability and providing a basis for controlling and eliminating diseases. Zamir et al. [8] established SIR mathematical models for humans, hosts, and media, using the Routh-Hurwitz standard and next-generation methods to obtain threshold conditions and give numerical simulation results. There are three main control strategies in the controls of VL model: parameter control strategy, optimal control strategy, and control strategy selection using simulation. However, the most generalized control strategy is the parameter control strategy. When the parameters are adjusted, people can apply it to a real-world control strategy, and there is also a lot of research on optimal control strategy and control strategy selection using simulation. Simulation comparison is the most common method of VL mathematic control modeling in simulation. It can compare the human infected population with control and without control. Meanwhile, it also proves the effectiveness of the combined control strategies [9]. With reference to these established mathematical models, we have established a mathematical model based on logistic process.

The purpose of this paper is to know the transmission state about VL among humans, dogs, and sandflies in the Kashgar Prefecture of Xinjiang. Firstly, we proposed a model to simulate the cumulative data of the Kashgar Prefecture and estimate the basic reproduction number and the dynamic behavior of the model. Then, sensitivity analysis was performed on the number of leishmaniasis human/dog/ sandfly cases and the basic reproduction number $R_{0}$ based on some key parameters. Finally, we explored some effective strategies for the prevention and control of VL in Kashgar.
The article is organized as follows. In Section 2, this paper introduces the model of VL and gives the expression of the basic reproduction number and the parameter value. The dynamical behaviors of the model are analyzed to better understand the transmission trends of the disease. Some mathematical analyses are given in Section 3. The numerical simulations, prediction of the epidemic trends for the next decades, estimation of the basic reproduction number, and sensitivity analysis of the basic reproduction number and the number of infected humans/sandflies/dogs are presented in Section 4. In Section 5, some brief summaries and discussion are given.

\section{Mathematical Model}

In order to study the transmission of VL in Kashgar, we developed a mathematical model based on humans, dogs, and sandflies, where the human population is divided into four groups: the susceptible, the exposed, the infected, and the recovered denoted by $S_{h}, E_{h}, I_{h}$, and $R_{h}$, respectively. The dog population is divided into two groups: the susceptible and the infected, denoted by $S_{r}$ and $I_{r}$, respectively. We divide the sandfly population into two subclasses: the susceptible and the infected, denoted by $S_{v}$ and $I_{v}$, respectively. And the total population for humans, dogs, and sandflies is $N_{h}=S_{h}+E_{h}+I_{h}+R_{h} ; \quad N_{v}=S_{v}+I_{v} ; \quad N_{r}=S_{r}+I_{r}$. The flowchart of VL transmission is illustrated in Figure 2.

The transmission process of VL is described by the following eight differential equations:

$$
\left\{\begin{array}{l}
\frac{\mathrm{d} S_{h}}{\mathrm{~d} t}=r_{1} S_{h}\left(1-\frac{S_{h}+E_{h}+I_{h}+R_{h}}{K_{1}}\right)-\beta_{1} S_{h} I_{v} \\
\frac{\mathrm{d} E_{h}}{\mathrm{~d} t}=\beta_{1} S_{h} I_{v}-\mu_{h} E_{h}-\omega E_{h} \\
\frac{\mathrm{d} I_{h}}{\mathrm{~d} t}=\omega E_{h}-\left(\mu_{h}+\delta_{1}\right) I_{h}-\gamma I_{h} \\
\frac{\mathrm{d} R_{h}}{\mathrm{~d} t}=\gamma_{h}-\mu_{h} R_{h} \\
\frac{\mathrm{d} S_{v}}{\mathrm{~d} t}=r_{2} S_{v}\left(1-\frac{S_{v}+I_{v}}{K_{2}}\right)-\left(\beta_{2} I_{h}+\beta_{3} I_{r}\right) S_{v} \\
\frac{\mathrm{d} I_{v}}{\mathrm{~d} t}=\left(\beta_{2} I_{h}+\beta_{3} I_{r}\right) S_{v}-\mu_{v} I_{v} \\
\frac{\mathrm{d} S_{r}}{\mathrm{~d} t}=r_{3} S_{r}\left(1-\frac{S_{r}+I_{r}}{K_{3}}\right)-\beta_{4} I_{v} S_{r} \\
\frac{\mathrm{d} I_{r}}{\mathrm{~d} t}=\beta_{4} I_{v} S_{r}-\left(\mu_{r}+\delta_{2}\right) I_{r} .
\end{array}\right.
$$

We assume that the susceptible individuals $S_{h}$ and dogs $S_{r}$ are bitten by infected sandflies with $\beta_{1}$ and $\beta_{4}$ infection 

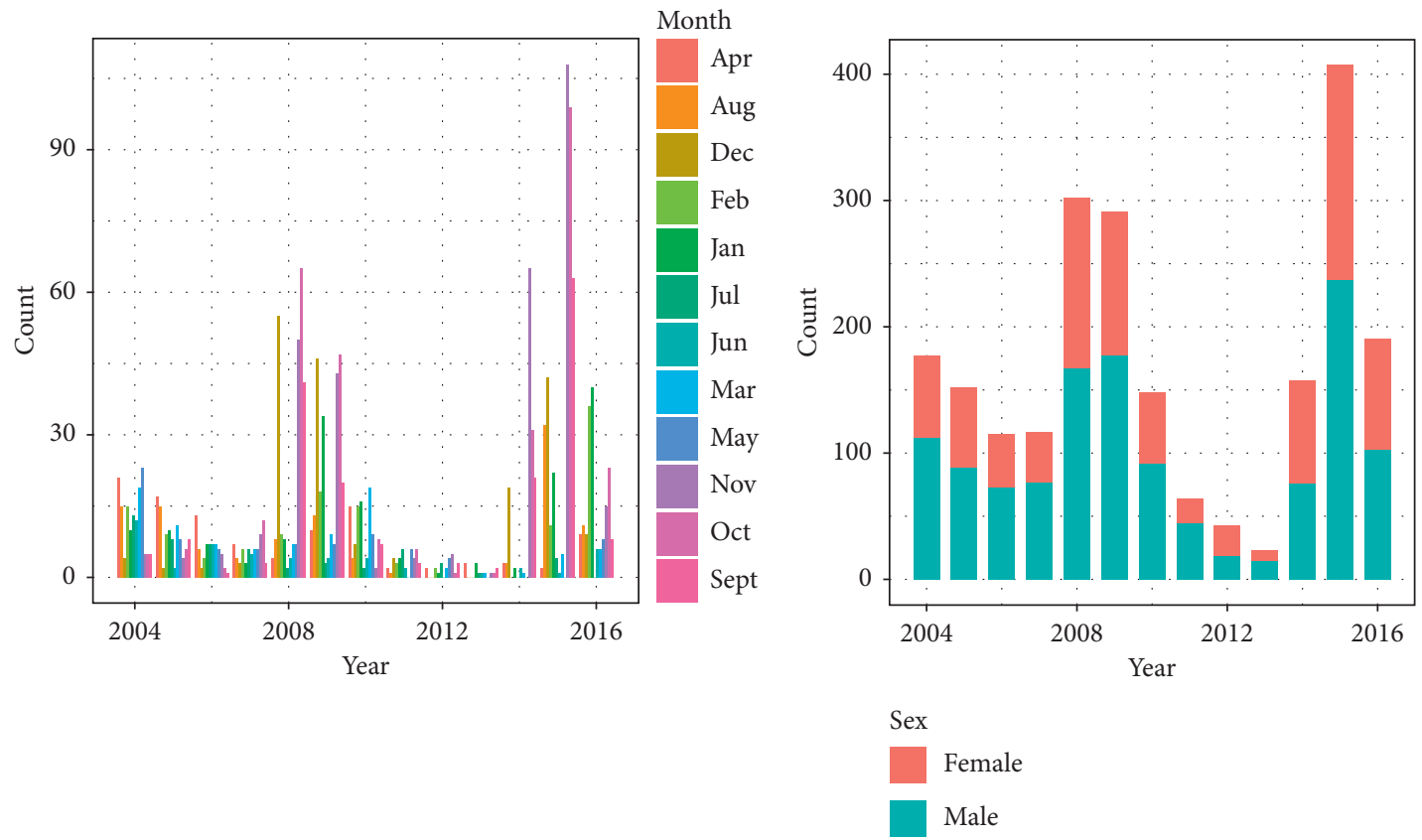

(a)

(b)

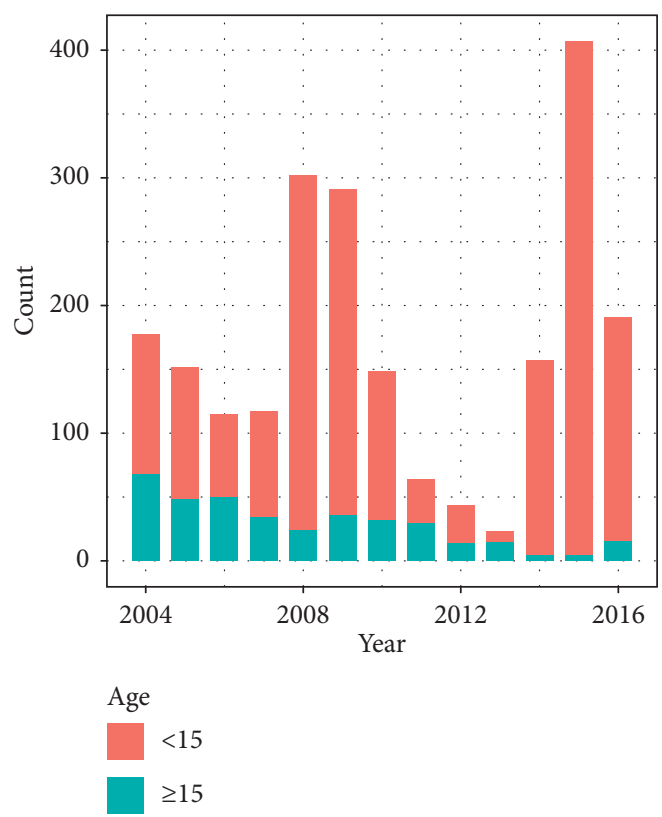

(c)

FIgURE 1: The data of human leishmaniasis cases from 2004 to 2016. (a) The reported data are described at different years. (b) The reported data are described at different genders and (c) different ages from 2004 to 2016.

rates, respectively. And the transmission probability from an infected dog or an infected individual to a susceptible sandfly is $\beta_{3}$ and $\beta_{2}$, respectively. $1 / \omega$ and $1 / \gamma$ indicate the latency and recovery rate of VL, respectively. The natural mortality rates of humans, vectors, and reserves are $\mu_{h}, \mu_{v}$, and $\mu_{r}$, respectively. The death rate of humans and reserves caused by VL are $\delta_{1}$ and $\delta_{2}$, respectively. The susceptible human population is governed by the logistic growth with carrying capacity $K_{1}$ as well as intrinsic growth rate $r_{1}$. In the absence of disease, the vector population density grows according to a logistic curve carrying capacity $K_{2}$, with an intrinsic growth rate $r_{2}$. In the absence of disease, the dog population density grows according to a logistic curve carrying capacity $K_{3}$, with an intrinsic growth rate $r_{3}$.

\section{Mathematical Analysis}

3.1. Basic Reproduction Number. The basic reproduction number $R_{0}$ refers to the number of people infected by a patient during the average period of illness when all people are susceptible. $R_{0}$ was calculated using the next-generation 


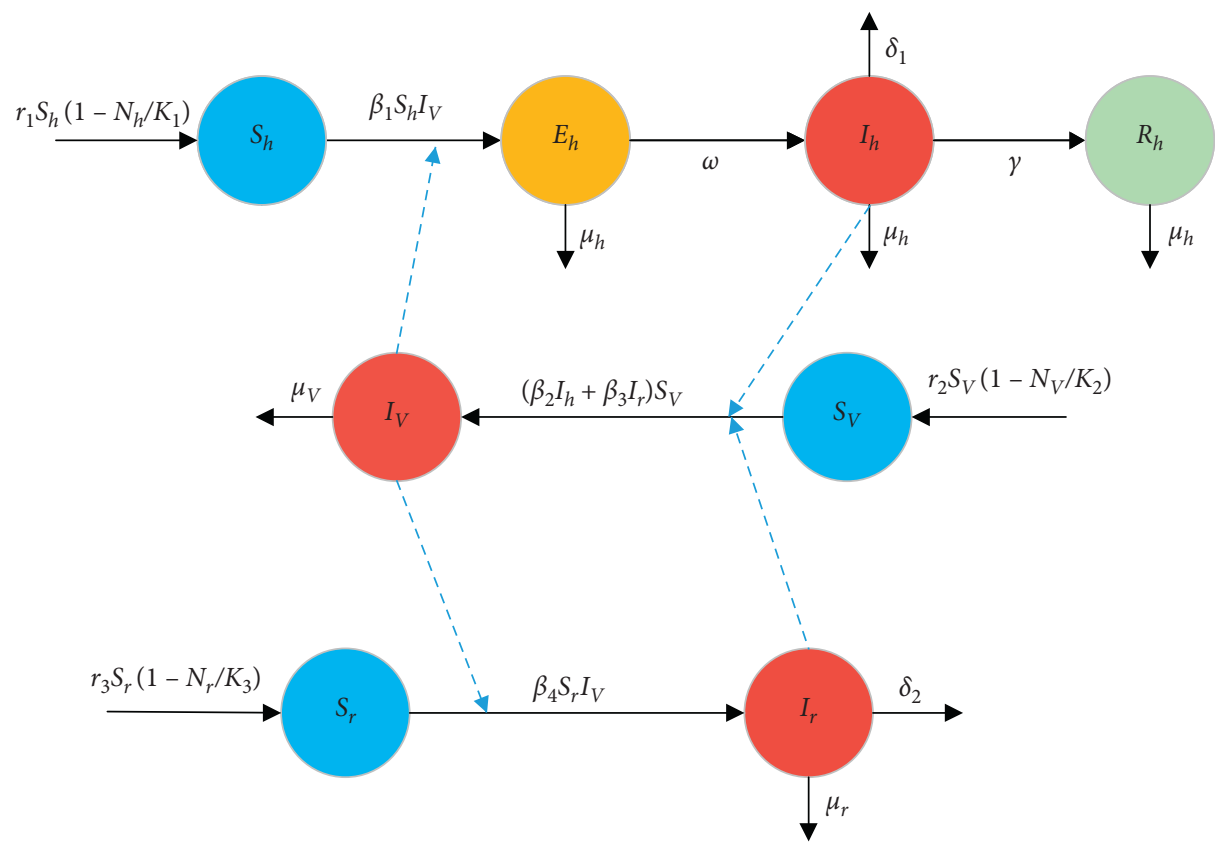

Figure 2: Flow diagram of the VL transmission model.

matrix $[15,16]$. The infected compartments are $E_{h}, I_{h}, I_{v}$, and $I_{r}$, giving $m=4$, so we have

$$
\begin{aligned}
& \mathscr{F}=\left[\begin{array}{c}
\beta_{1} S_{h} I_{v} \\
0 \\
\left(\beta_{2} I_{h}+\beta_{3} I_{r}\right) S_{v} \\
\beta_{4} I_{v} S_{r}
\end{array}\right], \\
& v=\left[\begin{array}{c}
\left(\mu_{h}+\omega\right) E_{h} \\
\left(\mu_{h}+\delta_{1}+\gamma\right) I_{h}-\omega E_{h} \\
\mu_{r} I_{r} \\
\left(\mu_{r}+\delta_{2}\right) I_{r}
\end{array}\right] .
\end{aligned}
$$

Thus,

$$
\mathbf{F V}^{-1}=\left[\begin{array}{cccc}
0 & 0 & \frac{\beta_{1} K_{1}}{\mu_{v}} & 0 \\
0 & 0 & 0 & 0 \\
\frac{\omega \beta_{2} K_{2}}{\left(\mu_{h}+\omega\right)\left(\mu_{h}+\delta_{1}+\gamma\right)} \frac{\beta_{2} K_{2}}{\mu_{h}+\delta_{1}+\gamma} & 0 & \frac{\beta_{3} K_{2}}{\mu_{r}+\delta_{2}} \\
0 & 0 & \frac{\beta_{4} K_{3}}{\mu_{v}} & 0
\end{array}\right] .
$$

Then,

$$
\begin{aligned}
F & =\left[\begin{array}{cccc}
0 & 0 & \beta_{1} K_{1} & 0 \\
0 & 0 & 0 & 0 \\
0 & \beta_{2} K_{2} & 0 & \beta_{3} K_{3} \\
0 & 0 & \beta_{4} K_{3} & 0
\end{array}\right], \\
V & =\left[\begin{array}{cccc}
\mu_{h}+\omega & 0 & 0 & 0 \\
-\omega & \mu_{h}+\delta_{1}+\gamma & 0 & 0 \\
0 & 0 & \mu_{v} & 0 \\
0 & 0 & 0 & \mu_{r}+\delta_{2}
\end{array}\right] .
\end{aligned}
$$

$$
R_{0}=\sqrt{\frac{K_{2}\left(K_{3} \beta_{3} \beta_{4}\left(\mu_{h}^{2}+\delta_{1} \mu_{h}+\gamma \mu_{h}+\delta_{1} \omega+\gamma \omega+\mu_{h} \omega\right)+\omega K_{1} \beta_{1} \beta_{2}\left(\delta_{2}+\mu_{r}\right)\right)}{\mu_{v}\left(\delta_{2}+\mu_{r}\right)\left(\mu_{h}+\omega\right)\left(\delta_{1}+\gamma+\mu_{h}\right)}} .
$$


$R_{0}$ can be used as a basic indicator in the study of VL propagation dynamics model. Usually, when $R_{0}=1$, it can be used as a threshold for the demise of the disease. When $R_{0}>1$, the disease will not die and eventually turn into endemic disease; when $R_{0}<1$, the disease will die out naturally.
3.2. Dynamic Behaviors for the Model. The dynamical behavior of model (1) is analyzed to better understand the transmission trends of the disease. We begin with elementary properties of solutions to the model (1). From biological considerations, we study model (1) in the set

$$
\begin{aligned}
& \Gamma=\left\{\left(S_{h}, E_{h}, I_{h}, R_{h}, S_{v}, I_{v}, S_{r}, I_{r}\right) \in \mathbb{R}_{+}^{8}: 0<S_{h} \leq K_{1}, 0<S_{v} \leq K_{2}, 0<S_{r} \leq K_{3}, N_{h} \leq \frac{\left(\mu_{h}+r_{1}\right)^{2} K_{1}}{4 \mu_{h} r_{1}}, N_{v} \leq \frac{\left(\mu_{v}+r_{2}\right)^{2} K_{2}}{4 \mu_{v} r_{2}},\right. \\
&\left.N_{r} \leq \frac{\left(\mu_{r}+r_{3}\right)^{2} K_{3}}{4 \mu_{r} r_{3}}\right\} .
\end{aligned}
$$

In fact, by using

$$
\begin{aligned}
\frac{\mathrm{d} S_{h}}{\mathrm{~d} t} & \leq r_{1} S_{h}\left(1-\frac{S_{h}}{K_{1}}\right), \\
\frac{\mathrm{d} S_{v}}{\mathrm{~d} t} & \leq r_{2} S_{v}\left(1-\frac{S_{v}}{K_{2}}\right), \\
\frac{\mathrm{d} S_{r}}{\mathrm{~d} t} & \leq r_{3} S_{r}\left(1-\frac{S_{r}}{K_{3}}\right),
\end{aligned}
$$

we can deduce that $S_{h}(t) \leq K_{1}, S_{v}(t) \leq K_{2}$, and $S_{r}(t) \leq K_{3}$ whenever $S_{h}(0) \leq K_{1}, S_{v}(0) \leq K_{2}$, and $S_{r}(0) \leq K_{3}$. Adding the first four equations of (1) yields

$$
\begin{aligned}
\frac{\mathrm{d} N_{h}}{\mathrm{~d} t} & =r_{1} S_{h}\left(1-\frac{N_{h}}{K_{1}}\right)-\mu_{h} N_{h}-\delta_{1} I_{h}+\mu_{h} S_{h} \\
& \leq\left(r_{1}+\mu_{h}\right) S_{h}-\frac{r_{1} S_{h}^{2}}{K_{1}}-\mu_{h} N_{h} \\
& \leq \frac{\left(r_{1}+\mu_{h}\right)^{2} K_{1}}{4 r_{1}}-\mu_{h} N_{h} .
\end{aligned}
$$

In view of system (8), it is easy to check that $N_{h}^{\prime}(t) \leq 0$ wherever $N_{h} \geq\left(\left(r_{1}+\mu_{h}\right)^{2} K_{1} / 4 \mu_{h} r_{1}\right)$. The same conclusion can be drawn for $N_{v}$ and $N_{r}$. According to the above analysis, $\Gamma$ is a maximum positive invariant set of (1). There always exists a disease-free equilibrium $P_{0}\left(K_{1}, 0,0,0, K_{2}, 0\right.$, $\left.K_{3}, 0\right)$. In the following, we will show the stability for the disease-free equilibrium.

Theorem 1. The disease-free equilibrium $P_{0}$ is locally asymptotically stable if $R_{0}<1$ and unstable if $R_{0}>1$.

Proof. The Jacobian matrix of model (1) at $P_{0}$ is given by

$$
J\left(P_{0}\right)=\left[\begin{array}{cccccccc}
-r_{1} & -r_{1} & -r_{1} & -r_{1} & 0 & -\beta_{1} K_{1} & 0 & 0 \\
0 & -\left(\mu_{h}+\omega\right) & 0 & 0 & 0 & \beta_{1} K_{1} & 0 & 0 \\
0 & \omega & -\left(\mu_{h}+\delta_{1}+\gamma\right) & 0 & 0 & 0 & 0 & 0 \\
0 & 0 & \gamma & -\mu_{h} & 0 & 0 & 0 & 0 \\
0 & 0 & -\beta_{2} K_{2} & 0 & -r_{2} & -r_{2} & 0 & -\beta_{3} K_{2} \\
0 & 0 & \beta_{2} K_{2} & 0 & 0 & -\mu_{v} & 0 & \beta_{3} K_{2} \\
0 & 0 & 0 & 0 & 0 & -\beta_{4} K_{3} & -r_{3} & -r_{3} \\
0 & 0 & 0 & 0 & 0 & \beta_{4} K_{3} & 0 & -\left(\mu_{r}+\delta_{2}\right)
\end{array}\right] .
$$

It is easy to see that there are four negative eigenvalues of $J\left(P_{0}\right):-r_{1},-r_{2},-r_{3},-\mu_{h}$. The other eigenvalues are determined by the following fourth-order equation:

$$
\lambda^{4}+a_{1} \lambda^{3}+a_{2} \lambda^{2}+a_{3} \lambda+a_{4}=0
$$

where 


$$
\begin{aligned}
a_{1}= & m_{1}+m_{2}+n+\mu_{v}, \\
a_{2}= & m_{1} m_{2}+\mu_{v} n-\beta_{3} \beta_{4} K_{2} K_{3}+\left(m_{1}+m_{2}\right)\left(\mu_{v}+n\right), \\
a_{3}= & m_{1} m_{2}\left(\mu_{v}+n\right)+\left(m_{1}+m_{2}\right)\left(\mu_{v} n-\beta_{3} \beta_{4} K_{2} K_{3}\right) \\
& -\omega \beta_{1} \beta_{2} K_{1} K_{2}, \\
a_{4}= & m_{1} m_{2}\left(\mu_{v} n-\beta_{3} \beta_{4} K_{2} K_{3}\right)-n \omega \beta_{1} \beta_{2} K_{1} K_{2},
\end{aligned}
$$

$$
\begin{aligned}
m_{1} & =\mu_{h}+\omega, \\
m_{2} & =\mu_{h}+\delta_{1}+\gamma, \\
n & =\mu_{r}+\delta_{2} .
\end{aligned}
$$

The inequality $R_{0}<1$ implies that

$$
\begin{gathered}
\beta_{3} \beta_{4} K_{2} K_{3}<\mu_{v} n, \\
\omega \beta_{1} \beta_{2} K_{1} K_{2}<m_{1} m_{2} \mu_{v}, \\
m_{1} m_{2} \beta_{3} \beta_{4} K_{2} K_{3}+n \omega \beta_{1} \beta_{2} K_{1} K_{2}<m_{1} m_{2} \mu_{v} n .
\end{gathered}
$$

From (13), we have $a_{i}>0$ for $i=1,2,3,4$. On account of the above inequalities, we have

$$
\begin{aligned}
a_{1} a_{2}-a_{3}= & \left.\left(m_{1}+m_{2}+n+\mu_{v}\right)\left[m_{1} m_{2}+\mu_{v} n-\beta_{3} \beta_{4} K_{2} K_{3}\right]+\left(m_{1}+m_{2}\right)\left(\mu_{v}+n\right)\right]-m_{1} m_{2}\left(\mu_{v}+n\right)-\left(m_{1}+m_{2}\right)\left(\mu_{v} n-\beta_{3} \beta_{4} K_{2} K_{3}\right) \\
& +\omega \beta_{1} \beta_{2} K_{1} K_{2} \\
= & m_{1} m_{2}\left(m_{1}+m_{2}\right)+\left(\mu_{v}+n\right)\left(\mu_{v} n-\beta_{3} \beta_{4} K_{2} K_{3}\right)+\left(m_{1}+m_{2}+n+\mu_{v}\right)\left(m_{1}+m_{2}\right)\left(\mu_{v}+n\right)+\omega \beta_{1} \beta_{2} K_{1} K_{2}>0 .
\end{aligned}
$$

Furthermore,

$$
\begin{aligned}
a_{3}\left(a_{1} a_{2}-a_{3}\right)-a_{1}^{2} a_{4}= & {\left[m_{1} m_{2}\left(\mu_{v}+n\right)+\left(m_{1}+m_{2}\right)\left(\mu_{v} n-\beta_{3} \beta_{4} K_{2} K_{3}\right)-\omega \beta_{1} \beta_{2} K_{1} K_{2}\right] } \\
& {\left[m_{1} m_{2}\left(m_{1}+m_{2}\right)+\left(\mu_{v}+n\right)\left(\mu_{v} n-\beta_{3} \beta_{4} K_{2} K_{3}\right)+\left(m_{1}+m_{2}+n+\mu_{v}\right)\left(m_{1}+m_{2}\right)\left(\mu_{v}+n\right)+\omega \beta_{1} \beta_{2} K_{1} K_{2}\right] } \\
& -m_{1} m_{2}\left(\mu_{v} n-\beta_{3} \beta_{4} K_{2} K_{3}\right)\left(m_{1}+m_{2}+n+\mu_{v}\right)^{2}+n \omega \beta_{1} \beta_{2} K_{1} K_{2}\left(m_{1}+m_{2}+n+\mu_{v}\right)^{2} \\
> & \left(m_{1}+m_{2}\right)\left(\mu_{v} n-\beta_{3} \beta_{4} K_{2} K_{3}\right)\left[m_{1} m_{2}\left(m_{1}+m_{2}\right)+\left(m_{1}+m_{2}+n+\mu_{v}\right)\left(m_{1}+m_{2}\right)\left(\mu_{v}+n\right)+\omega \beta_{1} \beta_{2} K_{1} K_{2}\right] \\
& -m_{1} m_{2}\left(\mu_{v} n-\beta_{3} \beta_{4} K_{2} K_{3}\right)\left(m_{1}+m_{2}+n+\mu_{v}\right)^{2} \\
= & \left(m_{1}^{2}+m_{2}^{2}\right)\left(\mu_{v} n-\beta_{3} \beta_{4} K_{2} K_{3}\right)\left(m_{1}+m_{2}\right)\left(\mu_{v}+n\right)+\left(m_{1}^{2}+m_{2}^{2}+m_{1} m_{2}\right)\left(\mu_{v} n-\beta_{3} \beta_{4} K_{2} K_{3}\right)\left(\mu_{v}+n\right)^{2}>0 .
\end{aligned}
$$

Therefore, each eigenvalue of equation (10) admits negative real part. When $R_{0}<1$, the disease-free equilibrium $P_{0}$ is locally asymptotically stable. When $R_{0}>1$, we conclude from $a_{4}>0$ that equation (10) has at least one positive real root; hence, the disease-free equilibrium $P_{0}$ is unstable.

Theorem 2. As $R_{0}<1$, the disease-free equilibrium is globally asymptotically stable in $\Gamma$.

Proof. Let us consider the following Lyapunov function:

$$
\begin{aligned}
V(t)= & \omega n \beta_{2} K_{2} E_{h}(t)+m_{1} n \beta_{2} K_{2} I_{h}(t)+m_{1} m_{2} n I_{v}(t) \\
& +m_{1} m_{2} \beta_{3} K_{2} I_{r}(t),
\end{aligned}
$$

where

$$
\begin{aligned}
m_{1} & =\mu_{h}+\omega, \\
m_{2} & =\mu_{h}+\delta_{1}+\gamma, \\
n & =\mu_{r}+\delta_{2} .
\end{aligned}
$$

The derivative of $V(t)$ along solutions of (1) is

$$
\begin{aligned}
\dot{V}(t)= & \omega n \beta_{1} \beta_{2} K_{2} S_{h} I_{v}-m_{1} m_{2} n \beta_{2} K_{2} I_{h}+m_{1} m_{2} n S_{v}\left(\beta_{2} I_{h}+\beta_{3} I_{r}\right) \\
& -m_{1} m_{2} \mu_{v} n I_{v}+m_{1} m_{2} \beta_{3} \beta_{4} K_{2} S_{r} I_{v}-m_{1} m_{2} n \beta_{3} K_{2} I_{r} \\
\leq & {\left[m_{1} m_{2} \beta_{3} \beta_{4} K_{2} K_{3}+n \omega \beta_{1} \beta_{2} K_{1} K_{2}-m_{1} m_{2} \mu_{v} n\right] I_{v} } \\
= & m_{1} m_{2} \mu_{v} n\left(R_{0}^{2}-1\right) I_{v} \\
\leq & 0 .
\end{aligned}
$$

By LaSalle's invariance principle, the omega limit set of each solution starting from $\Gamma$ lies in an invariant set contained in 


$$
\Omega=\left\{\left(S_{h}, E_{h}, I_{h}, R_{h}, S_{v}, I_{v}, S_{r}, I_{r}\right) \in \Gamma: I_{v}=0\right\} .
$$

It can be verified that the only invariant set contained in $\Omega$ is the singleton $\left\{P_{0}\right\}$. Then, the disease-free equilibrium $P_{0}$ is globally asymptotically stable. This completes the proof. In the following, we will consider the dynamical behavior of model (1) when $R_{0}>1$.

Theorem 3. For any solution $\left(S_{h}(t), E_{h}(t), I_{h}(t), R_{h}(t)\right.$, $\left.S_{v}(t), I_{v}(t), S_{r}(t), I_{r}(t)\right)$ of (1) with initial values $S_{h}(0)>0$, $E_{h}(0) \geq 0, I_{h}(0) \geq 0, R_{h}(0) \geq 0, S_{v}(0)>0, I_{v}(0) \geq 0, S_{r}(0)>0$, $I_{r}(0) \geq 0$, and $I_{h}(0)+I_{v}(0)+I_{r}(0)>0$, there exists $v>0$ such that

$$
\begin{aligned}
& \liminf _{t \longrightarrow \infty} I_{h}(t)>v, \\
& \liminf _{t \longrightarrow \infty} I_{v}(t)>v, \\
& \liminf _{t \longrightarrow \infty} I_{r}(t)>v,
\end{aligned}
$$

as $R_{0}>1$.

$M_{\partial}=\left\{\left(S_{h}(0), E_{h}(0), I_{h}(0), R_{h}(0), S_{v}(0), I_{v}(0), S_{r}(0), I_{r}(0)\right):\right.$

: $\left.\left(S_{h}(t), E_{h}(t), I_{h}(t), R_{h}(t), S_{v}(t), I_{v}(t), S_{r}(t), I_{r}(t)\right) \in \partial X_{0}, t \geq 0\right\}$.

We first claim that

$$
M_{\partial}=\left\{\left(S_{h}, 0,0, R_{h}, S_{v}, 0, S_{r}, 0\right): S_{h}, R_{h}, S_{v}, S_{r} \geq 0\right\} .
$$

Suppose that $\left(S_{h}(0), E_{h}(0), I_{h}(0), R_{h}(0), S_{v}(0), I_{v}(0)\right.$, $\left.S_{r}(0), I_{r}(0)\right) \in M_{\partial}$. It suffices to show $E_{h}(t)=0$ for all $t \geq 0$. If it is not true, then there exists $t_{0}>0$ such that $E_{h}\left(t_{0}\right)>0$. Then, $E_{h}^{\prime}(t) \geq\left(\mu_{h}+\omega\right) E_{h}(g)$ implies $E_{h}(t)>0$ for all $t \geq t_{0}$. From the third equation of (1), it may be concluded that

$$
\begin{aligned}
I_{h}(t) & =e^{-\left(\mu_{h}+\delta_{1}+\gamma\right)\left(t-t_{0}\right)} I_{h}\left(t_{0}\right)+\int_{t_{0}}^{t} e^{-\left(\mu_{h}+\delta_{1}+\gamma\right)(t-\theta)} \omega E_{h}(\theta) \mathrm{d} \theta \\
& \geq \int_{t_{0}}^{t} e^{-\left(\mu_{h}+\delta_{1}+\gamma\right)(t-\theta)} \omega E_{h}(\theta) \mathrm{d} \theta>0,
\end{aligned}
$$

for all $t>t_{0}$. This is a contradiction with

$$
\left(S_{h}(0), E_{h}(0), I_{h}(0), R_{h}(0), S_{v}(0), I_{v}(0), S_{r}(0), I_{r}(0)\right) \in M_{\partial} .
$$

Thus, (23) is valid.

Denote

$$
\begin{aligned}
\Omega= & \bigcup \omega\left(S_{h}(0), E_{h}(0), I_{h}(0), R_{h}(0), S_{v}(0), I_{v}(0), S_{r}(0), I_{r}(0)\right): \\
& \left.\left(S_{h}(0), E_{h}(0), I_{h}(0), R_{h}(0), S_{v}(0), I_{v}(0), S_{r}(0), I_{r}(0)\right) \in M_{\partial}\right\},
\end{aligned}
$$

where $\quad \omega\left(S_{h}(0), E_{h}(0), I_{h}(0), R_{h}(0), S_{v}(0), I_{v}(0), S_{r}(0), I_{r}\right.$ (0)) is the omega limit set of the solution to (1) through $\left(S_{h}(0), E_{h}(0), I_{h}(0), R_{h}(0), S_{v}(0), I_{v}(0), S_{r}(0), I_{r}(0)\right)$.

Restricting (1) on $M_{\partial}$ yields
Proof. Let

Next, we will show that system (1) is uniformly persistent with respect to $\left(X_{0}, \partial X_{0}\right)$. Obviously, $X$ is positively invariant with respect to (1). Set $a=\max \left\{\mu_{h}+\delta_{1}+\right.$ $\left.\gamma, \mu_{v}, \mu_{r}+\delta_{2}\right\}$. The inequality $\left(I_{h}(t)+I_{v}(t)+I_{r}(t)\right)^{\prime} \geq$ $-a\left(I_{h}(t)+I_{v}(t)+I_{r}(t)\right)$ and $I_{h}(0)+I_{v}(0)+I_{r}(0)>0$ imply $I_{h}(t)+I_{v}(t)+I_{r}(t)>0$ for all $t \geq 0$ as $I_{h}(0)+$ $I_{v}(0)+I_{r}(0)>0$. Therefore, $X_{0}$ is also a positive invariant set for system (1). Furthermore, by Theorem 3, there exists a compact set $c$ in which all solutions of (1) initiated in $X$ will enter and remain forever after. The compactness condition $\left(C_{4.2}\right)$ in Thieme [17] is easily verified for this set $c$. Denote

$$
\begin{aligned}
X & =\left\{\left(S_{h}, E_{h}, I_{h}, R_{h}, S_{v}, I_{v}, S_{r}, I_{r}\right) \in \mathbb{R}_{+}^{8}: S_{h}>0, S_{v}>0, S_{r}>0\right\}, \\
X_{0} & =\left\{\left(S_{h}, E_{h}, I_{h}, R_{h}, S_{v}, I_{v}, S_{r}, I_{r}\right) \in X: I_{h}+I_{v}+I_{r}>0\right\}, \\
\partial X_{0} & =X \backslash X_{0} .
\end{aligned}
$$

$$
\left\{\begin{array}{l}
\frac{\mathrm{d} S_{h}}{\mathrm{~d} t}=r_{1} S_{h}\left(1-\frac{S_{h}+R_{h}}{K_{1}}\right) \\
\frac{\mathrm{d} R_{h}}{\mathrm{~d} t}=-\mu_{h} R_{h} \\
\frac{\mathrm{d} S_{v}}{\mathrm{~d} t}=r_{2} S_{v}\left(1-\frac{S_{v}}{K_{2}}\right) \\
\frac{\mathrm{d} S_{r}}{\mathrm{~d} t}=r_{3} S_{r}\left(1-\frac{S_{r}}{K_{3}}\right)
\end{array}\right.
$$

A trivial verification shows that system (27) has a unique equilibrium $\left(K_{1}, 0, K_{2}, K_{3}\right)$. Thus, $P_{0}\left(K_{1}, 0,0,0, K_{2}, 0, K_{3}, 0\right)$ is the unique equilibrium of (1) in $M_{\partial}$. It is easily seen that $\left(K_{1}, 0, K_{2}, K_{3}\right)$ is globally asymptotically stable. Therefore, we have $\Omega=\left\{P_{0}\right\}$. And $P_{0}$ is a covering of $\Omega$, which is isolated and is acyclic (since there exists no solution in $M_{\partial}$ which links $P_{0}$ to itself). Finally, the proof will be done if $P_{0}$ is a weak repeller for $X_{0}$, i.e.,

$$
\underset{t \longrightarrow \infty}{\limsup } \operatorname{dist}\left(\Psi(t), P_{0}\right)>0,
$$

where $\Psi(t)=\left(S_{h}(t), E_{h}(t), I_{h}(t), R_{h}(t), S_{v}(t), I_{v}(t), S_{r}(t)\right.$, $\left.I_{r}(t)\right)$ is an arbitrary solution with initial value in $X_{0}$. By Leenheer and Smith (Proof of Lemma 3.5, [18]), we only need to prove that $W^{s}\left(P_{0}\right) \cap X_{0}=\phi$ where $W^{s}\left(P_{0}\right)$ is the stable manifold of $E_{0}$. Suppose it is not true, then there exists a solution $\left(S_{h}(t), E_{h}(t), I_{h}(t), R_{h}(t), S_{v}(t), I_{v}(t), S_{r}(t)\right.$, $\left.I_{r}(t)\right)$ in $X_{0}$, such that as $t \longrightarrow \infty$, 


$$
\begin{aligned}
& S_{h}(t) \longrightarrow K_{1}, E_{h}(t) \longrightarrow 0, I_{h}(t) \longrightarrow 0, R_{h}(t) \longrightarrow 0, \\
& S_{v}(t) \longrightarrow K_{2}, I_{v}(t) \longrightarrow 0, S_{r}(t) \longrightarrow K_{3}, I_{r}(t) \longrightarrow 0 .
\end{aligned}
$$

When $R_{0}>1$,

$\omega \beta_{2} K_{2}\left(\mu_{r}+\delta_{2}\right) \beta_{1} K_{1}+\beta_{3} K_{2}\left(\mu_{h}+\omega\right)\left(\mu_{h}+\delta_{1}+\gamma\right) \beta_{4} K_{3}$

$>\left(\mu_{h}+\omega\right)\left(\mu_{h}+\delta_{1}+\gamma\right)\left(\mu_{r}+\delta_{2}\right) \mu_{v}$.

On account of (30), we may choose $\rho>0, \sigma>0, \eta>0$, and $\epsilon>0$ such that

$$
\begin{cases}0, & <\omega \beta_{2}\left(K_{2}-\epsilon\right)\left(\mu_{r}+\delta_{2}\right)-\rho \ll 1, \\ 0, & <\beta_{3}\left(K_{2}-\epsilon\right)\left(\mu_{r}+\omega\right)\left(\mu_{h}+\delta_{1}+\gamma\right)-\sigma \ll 1, \\ 0, & <\eta-\left(\mu_{h}+\omega\right)\left(\mu_{h}+\delta_{1}+\gamma\right)\left(\mu_{r}+\delta_{2}\right) \ll 1 .\end{cases}
$$

$$
\rho \beta_{1}\left(K_{1}-\epsilon\right)+\sigma \beta_{4}\left(K_{3}-\epsilon\right)-\eta \mu_{v}>0 .
$$

From (31), we see that $\left(\left(\mu_{h}+\omega\right) / \omega\right) \rho<\left(\mu_{h}+\omega\right)\left(\mu_{r}+\delta_{2}\right)$ $\beta_{2}\left(K_{2}-\epsilon\right)<\left(\beta_{2}\left(K_{2}-\epsilon\right)\right) /\left(\mu_{h}+\delta_{1}+\gamma\right) \eta$, $\eta \beta_{3}\left(K_{2}-\epsilon\right)>\sigma\left(\mu_{r}+\delta_{2}\right)$. Therefore, there exists $\xi$ such that

$$
\frac{\mu_{h}+\omega}{\omega} \rho<\xi<\frac{\beta_{2}\left(K_{2}-\epsilon\right)}{\mu_{h}+\delta_{1}+\gamma} \eta \text {. }
$$

For $\epsilon>0$, by (29), there exists $T>0$ such that

$$
\begin{aligned}
& K_{1}-\epsilon<S_{h}(t)<K_{1}+\epsilon, \\
& K_{2}-\epsilon<S_{v}(t)<K_{2}+\epsilon, \\
& K_{3}-\epsilon<S_{r}(t)<K_{3}+\epsilon,
\end{aligned}
$$

for all $t \geq T$. Let

$$
L(t)=\rho E_{h}(t)+\xi I_{h}(t)+\eta I_{v}(t)+\sigma I_{r}(t) .
$$

The derivative of $L$ along the solution $\left(S_{h}(t), E_{h}(t)\right.$, $\left.I_{h}(t), R_{h}(t), S_{v}(t), I_{v}(t), S_{r}(t), I_{r}(t)\right)$ is given by

$$
\begin{aligned}
L^{\prime}(t)= & {\left[\xi \omega-\rho\left(\mu_{h}+\omega\right)\right] E_{h}+\left[\eta \beta_{2} S_{v}-\xi\left(\mu_{h}+\delta_{1}+\gamma\right)\right] I_{h}+\left[\rho \beta_{1} S_{h}+\sigma \beta_{4} S_{r}-\eta \mu_{v}\right] I_{v}+\left[\eta \beta_{3} S_{v}-\sigma\left(\mu_{r}+\delta_{2}\right)\right] I_{r} } \\
\geq & {\left[\xi \omega-\rho\left(\mu_{h}+\omega\right)\right] E_{h}+\left[\eta \beta_{2}\left(K_{2}-\epsilon\right)-\xi\left(\mu_{h}+\delta_{1}+\gamma\right)\right] I_{h}+\left[\rho \beta_{1}\left(K_{1}-\epsilon\right)+\sigma \beta_{4}\left(K_{3}-\epsilon\right)-\eta \mu_{v}\right] I_{v} } \\
& +\left[\eta \beta_{3}\left(K_{2}-\epsilon\right)-\sigma\left(\mu_{r}+\delta_{2}\right)\right] I_{r} \\
\geq & \varrho L(t),
\end{aligned}
$$

for all $t \geq T$, where

$$
\varrho=\min \left\{\frac{\xi \omega-\rho\left(\mu_{h}+\omega\right)}{\rho}, \frac{\eta \beta_{2}\left(K_{2}-\epsilon\right)-\xi\left(\mu_{h}+\delta_{1}+\gamma\right)}{\xi}, \frac{\rho \beta_{1}\left(K_{1}-\epsilon\right)+\sigma \beta_{4}\left(K_{3}-\epsilon\right)-\eta \mu_{v}}{\eta}, \frac{\eta \beta_{3}\left(K_{2}-\epsilon\right)-\sigma\left(\mu_{r}+\delta_{2}\right)}{\sigma}\right\}>0 .
$$

Hence, $L(t) \longrightarrow \infty$ as $t \longrightarrow \infty$, which contradicts to the boundedness of $L(t)$. This completes the proof.

Remark 1. Theorems 1 3 state that the basic reproduction number $R_{0}$ is a sharp threshold value for model (1). As $R_{0}<1$, the disease-free equilibrium $P_{0}$ is globally stable, i.e., the disease will go to extinction. As $R_{0}>1$, the disease is uniformly persistent, i.e., the disease will become an endemic in the meaning of persistence.

\section{Results}

We applied model (1) to study the infection status of VL in Kashgar, Xinjiang. Most of the parameters were obtained from the literature, and some of them were assumed or simulated to have more realistic results. These parameter values are listed in Table 1 . The interpretation of the parameters values are as follows:
(1) According to the population statistics in Kashgar Prefecture in Xinjiang Statistical Yearbook 2004-2016 [20], we have estimated the carrying capacity of population $K_{1}=5000000$.

(2) According to the terrain and climate factors of Kashgar and the survival habits of the sandflies [21-24], the carrying capacity of sandflies $K_{2}=7000000$ has been estimated in Kashgar. In 2015, the number of dogs in Kashgar Prefecture of Xinjiang was close to 80,000 [25], so we have assumed the carrying capacity of dogs in Xinjiang $K_{3}=100000$.

(3) The values of the parameter $r_{1}$ are obtained by numerical differentiation according to the population data in Kashgar, and the value of $r_{2}$ is calculated according to the life cycle of the sandflies (see [19]); the value of $r_{3}$ is assumed because there is no the specific data about dog. 
TABle 1: Parameter values (and their sources).

\begin{tabular}{|c|c|c|c|}
\hline Parameters & Value & Interpretation* & Source \\
\hline$r_{1}$ & 0.019 & Intrinsic growth rate of human & Calculated \\
\hline$r_{2}$ & 0.026 & Intrinsic growth rate of vector & {$[19]$} \\
\hline$r_{3}$ & 0.296 & Intrinsic growth rate of reserve & Assumption \\
\hline$K_{1}$ & 5000000 & Human carrying capacity & Assumption \\
\hline$K_{2}$ & 7000000 & Vector carrying capacity & Assumption \\
\hline$K_{3}$ & 100000 & Reserve carrying capacity & Assumption \\
\hline$\beta_{1}$ & $1.35 e-08$ & Transmission probability from an infected sandfly to a susceptible human & Fitting \\
\hline$\beta_{2}$ & $3.565 e-17$ & Transmission probability from an infected human to a susceptible sand fly & Fitting \\
\hline$\beta_{3}$ & $3.515 e-05$ & Transmission probability from an infected reserve to a susceptible sandfly & Fitting \\
\hline$\beta_{4}$ & $1.23 e-05$ & Transmission probability from an infected sandfly to a susceptible reserve & Fitting \\
\hline$\mu_{h}$ & 0.0125 & Natural mortality rate of human & {$[10]$} \\
\hline$\mu_{v}$ & 25.55 & Natural mortality rate of vector & {$[11]$} \\
\hline$\mu_{r}$ & 0.1 & Natural mortality rate of reserve & [12] \\
\hline $1 / \omega$ & 0.33 & Human incubation period & [13] \\
\hline$\delta_{1}$ & 4.015 & VL-induced death rate of humans & {$[8]$} \\
\hline$\delta_{2}$ & 3.65 & VL-induced death rate of reserve & [13] \\
\hline $1 / \gamma$ & 2.5 & Recovery period of VL & {$[14]$} \\
\hline
\end{tabular}

We model cumulative cases as a Poisson-distributed random variable because the Poisson distribution describes the number of observed events in an interval of time. We calibrate the model by sampling from the posterior distribution of parameter vector $\theta\left|\mathbf{y}=\left\{\beta_{1}, \beta_{2}, \beta_{3}, \beta_{4}\right\}\right| \mathbf{y}$, where vector $\mathbf{y}$ is derived from $(\mathrm{d} / \mathrm{d} t) Y(t)=\omega E_{h}$ and $Y(t)$ denotes the reported cumulative cases. We conduct sampling via MCMC (Markov Chain Monte Carlo) using the Metropolis-Hastings acceptance rule. The posterior density is

$$
f_{\Theta \mid \mathbf{y}}(\theta \mid \mathbf{y})=\prod_{T} \mathscr{L}(Y(t) \mid \theta) f_{\Theta}(\theta) .
$$

The prior density $f_{\Theta}(\theta)$ is the joint probability of four univariate priors. We consider that $\beta_{1}, \beta_{2}, \beta_{3}$, and $\beta_{4}$ are distributed according to $u(0,1)$. The program was implemented in $\mathrm{R}$ version 3.6.0. We sampled from 30,000 MCMC iterations and discarded the first 10,000 samples as a burn-in period. On the basis of these 20,000 samples, the point estimates and 95\% confidence intervals for the transmission coefficients were calculated. The results are shown in Table 2.

The MCMC method was used to fit the cumulative incidence data of VL in Kashgar from 2004 to 2016, and the $95 \%$ confidence interval of the fitted curve was obtained (see Figure 3). It can be seen from the figure that the fitted values of the model matches are in accordance with the accumulated data values in the Kashgar Prefecture. Only some data fluctuate, but in any case, the cumulative data of VL in Kashgar are increasing year by year. This model is used to predict the prevalence of VL in the Kashgar Prefecture over the next decade (see Figure 4).

The basic reproduction number $R_{0}=1.76(95 \%$ CI: 1.49-1.93). The result shows that $R_{0}>1$; according to the threshold theory, the disease will not disappear in the Kashgar Prefecture in a short period of time, and an endemic disease will be formed.

It is well known that the basic reproduction number $R_{0}$ is a very important parameter in the infectious disease model. In our model, $R_{0}$ is determined by the parameters of $\omega, \delta_{1}$, $\delta_{2}, \gamma, \mu_{h}, \mu_{r}, \mu_{v}, \beta_{1}, \beta_{2}, \beta_{3}$, and $\beta_{4}$. We use the Latin hypercube
TABle 2: Parameter values for point estimation and 95\% interval estimation.

\begin{tabular}{lcc}
\hline Parameters & Point estimation & $95 \%$ confidence interval \\
\hline$\beta_{1}$ & $1.35 e-08$ & {$[1.06 e-08,1.94 e-08]$} \\
$\beta_{2}$ & $3.565 e-17$ & {$[2.87 e-19,2.44 e-15]$} \\
$\beta_{3}$ & $3.515 e-05$ & {$[2.067 e-05,1.002 e-04]$} \\
$\beta_{4}$ & $1.23 e-05$ & {$[3.18 e-06,2.36 e-05]$} \\
\hline
\end{tabular}

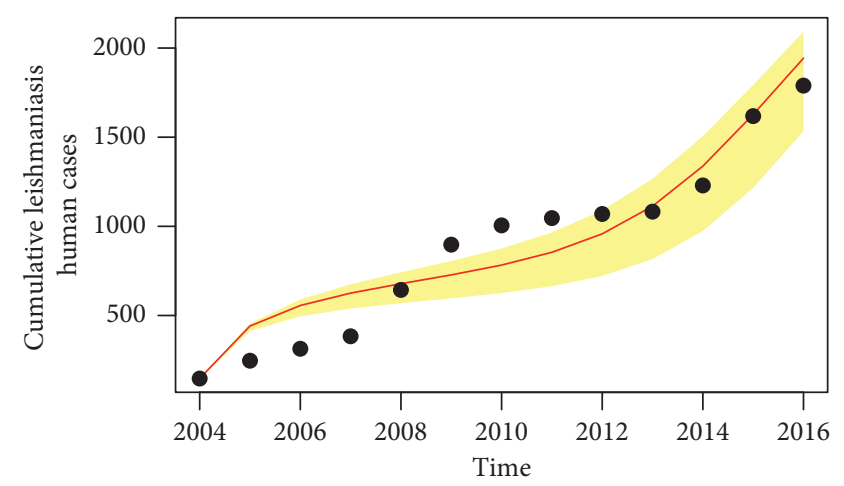

FIgUre 3: Cumulative incidence simulation of VL in Kashgar from 2004 to 2016

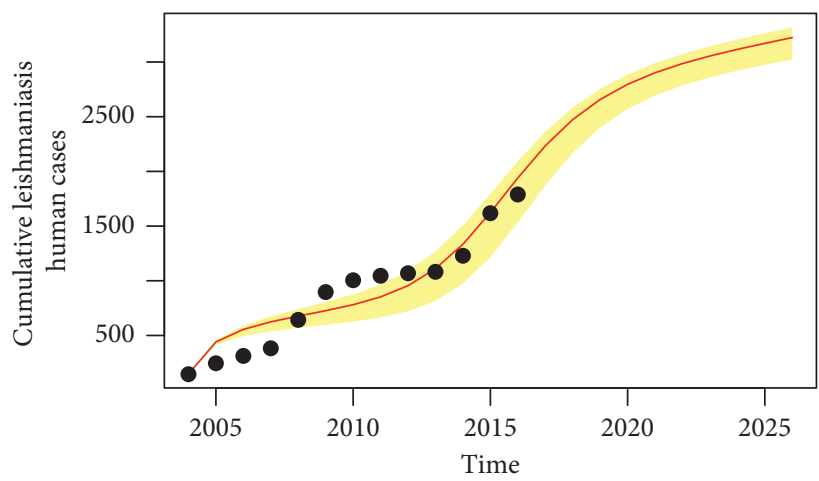

FIgURE 4: Prediction of cumulative incidence in 10 years from 2016 to 2026 . 
TABLE 3: Partial rank correlation coefficients (PRCCs) for $R_{0}$ on each input parameter variable.

\begin{tabular}{lcccc}
\hline Parameters & Distribution & \multicolumn{2}{c}{$\begin{array}{c}3000 \text { samples } \\
\text { samples }\end{array}$} & \multicolumn{2}{c}{$p$ value } \\
\hline$\omega$ & $N\left(3.03,0.15^{2}\right)$ & $9.295 e-04$ & 0.504 & $4.379 e-04$ \\
$\delta_{1}$ & $N\left(4.015,0.2^{2}\right)$ & $-1.995 e-04$ & 0.493 & $-1.619 e-04$ \\
$\delta_{2}$ & $N\left(3.65,0.2^{2}\right)$ & -0.247 & $<0.001$ & -0.2474 \\
$\gamma$ & $N\left(0.4,0.02^{2}\right)$ & $-1.281 e-03$ & 0.493 & $3.011 e-04$ \\
$\mu_{h}$ & $N\left(0.0125,0.0006^{2}\right)$ & $8.376 e-04$ & 0.511 & $-1.041 e-03$ \\
$\mu_{r}$ & $N\left(0.1,0.005^{2}\right)$ & $-6.862 e-03$ & 0.481 & $-6.146 e-03$ \\
$\mu_{v}$ & $N\left(25.55,3.28^{2}\right)$ & -0.2327 & $<0.001$ & -0.2334 \\
$\beta_{1}$ & $N(1.35 e-08,1 e-08)$ & $3.792 e-04$ & 0.506 & $-5.341 e-05$ \\
$\beta_{2}$ & $N\left(3.565 e-08,2 e-17^{2}\right)$ & $-3.859 e-04$ & 0.493 & $-3.456 e-04$ \\
$\beta_{3}$ & $N\left(3.515 e-08,1.5 e-05^{2}\right)$ & 0.914 & $<0.001$ & 0.495 \\
$\beta_{4}$ & $N\left(1.23 e-08,5 e-06^{2}\right)$ & 0.906 & $<0.001$ & 0.014 \\
\hline
\end{tabular}

sampling (LHS) and partial rank correlation coefficients (PRCCs) to examine parameters which have a significant influence on the transmission of VL [26]. Using model (1), 2000 and 3000 samples are randomly generated by assuming a uniform distribution for each parameter based on values from Table 1.

We select eleven parameters as input variables and calculate the corresponding PRCC and $p$ values for the eleven parameters. Table 3 and Figure 5 show the exact PRCC and $p$ values of each input parameter and the effect on the basic reproduction number $R_{0}$, respectively. We assume the significance level $\alpha=0.05$. The larger the absolute value of PRCC, the stronger the correlation between the input parameters and $R_{0}$. It can be seen from Table 3 that only $\delta_{2}$, $\mu_{v}, \beta_{3}$, and $\beta_{4}$ have significant impact on $R_{0}$. More concretely, parameters $\delta_{2}$ and $\mu_{v}$ have negative impact on $R_{0}$ and parameters $\beta_{3}$ and $\beta_{4}$ have positive impact.

We perform sensitivity analysis of infected humans, infected sandflies, and infected dogs through evaluating the PRCCs with the parameters of interest of model (1) over time by choosing a normal distribution with mean value and standard deviation shown in Figure 6. In Figures 6(a)-6(c), we plot the PRCCs over time with respect to the infected humans, sandflies, and infected dogs, respectively. Figure 6(a) indicates that there are three PRCC values that are significantly different from zero. The first three parameters with most impact on the outcome (the number of infected humans) are the sandfly-to-human transmission $\left(\beta_{1}\right)$, transmission from an infected dog to a susceptible sandfly $\left(\beta_{3}\right)$, and transmission from an infected sandfly to a susceptible $\operatorname{dog}\left(\beta_{4}\right)$. Figure 6(c) indicates that there are two PRCC values that are significantly different from zero. The first two parameters with most impact on the outcome (the number of infected humans) are the transmission from an infected dog to a susceptible sandfly $\left(\beta_{3}\right)$ and transmission from an infected sandfly to a susceptible dog $\left(\beta_{4}\right)$. Figure $6(c)$ indicates that there are two PRCC values that are significantly different from zero. The first two parameters with most impact on the outcome (the number of infected humans) are the transmission from an infected sandfly to a susceptible $\operatorname{dog}\left(\beta_{4}\right)$ and transmission from an infected dog to a susceptible sandfly $\left(\beta_{3}\right)$.

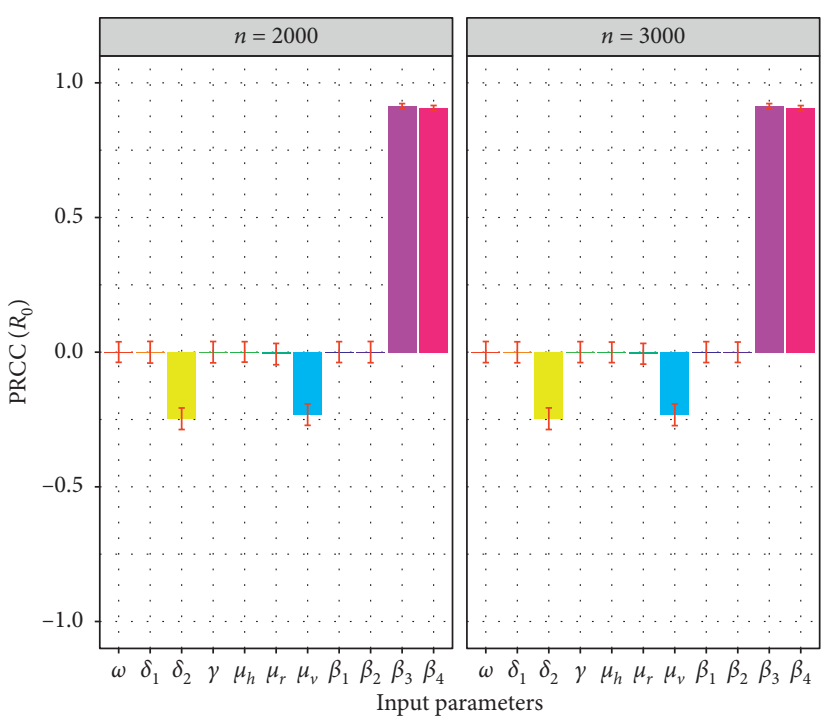

FIGURE 5: Partial rank correlation coefficient (PRCC) results for the dependence of $R_{0}$ on each parameter.

Through sensitivity analysis, we demonstrate that the infection rate from infected dogs to susceptible sandflies $\beta_{3}$ and infected sandflies to susceptible $\operatorname{dogs} \beta_{4}$ is the most sensitive parameter of $R_{0}$. And $\beta_{1}, \beta_{3}$, and $\beta_{4}$ are the most sensitive parameters for the number of leishmaniasis human cases. Therefore, it is necessary to change the values of the parameters to observe their effects on the number of leishmaniasis human cases and $R_{0}$ (see Figure 7). From Figure 7(a), we observe that the number of leishmaniasis human cases decrease with the decrease of $\beta_{1}$. We change the values of $\beta_{3}$ and $\beta_{4}$ to $4,2,1 / 2$, and $1 / 4$ times the original values. We observe the effects of different values on the number of leishmaniasis human cases, and we find that the change of parameter $\beta_{3}, \beta_{4}$ can influence not only the number of leishmaniasis human cases, but also the peak time. As Figures $7(\mathrm{~b})$ and 7 (c) illustrate, when fixing other parameters at constant, the number of leishmaniasis human cases fall with the decrease of $\beta_{3}$ and $\beta_{4}$, respectively. And the peak of outbreak will be postponed. 


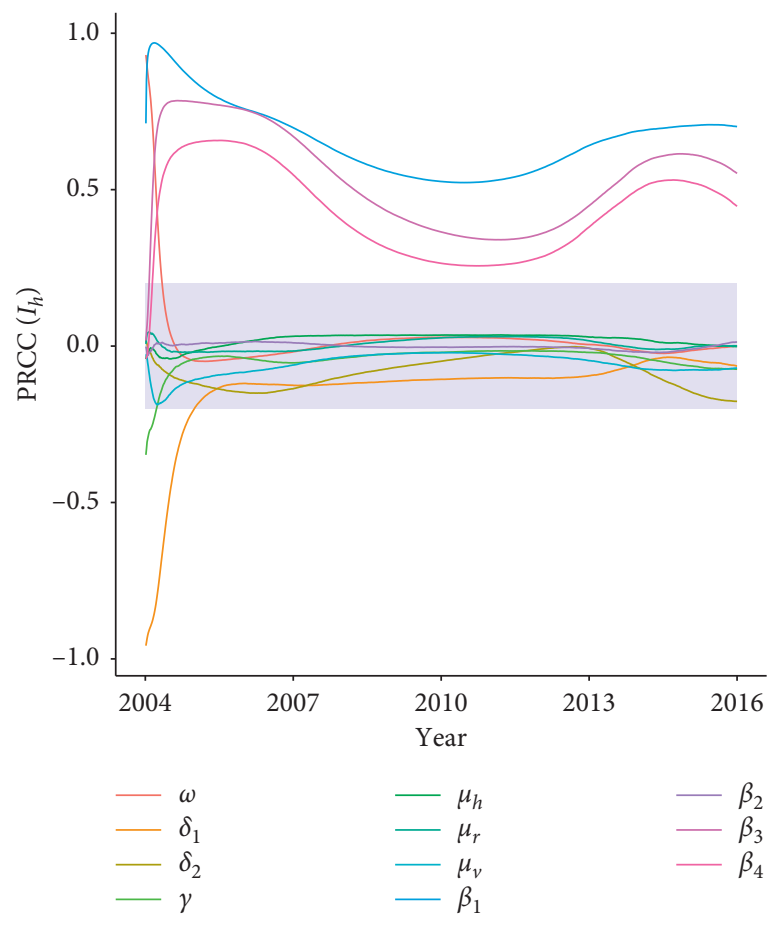

(a)

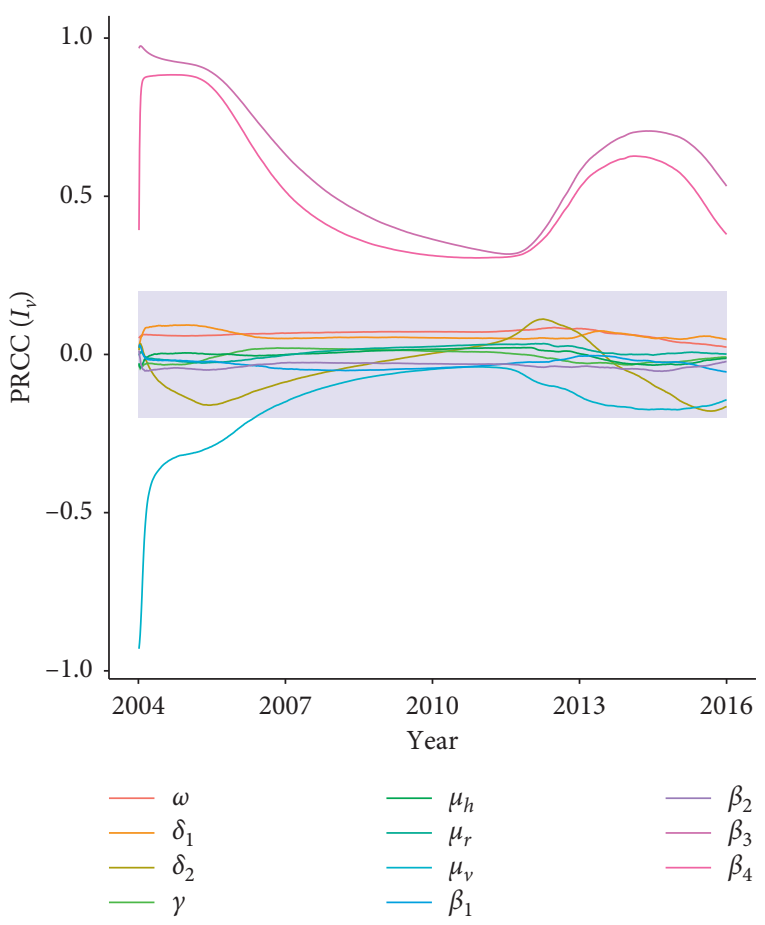

(b)

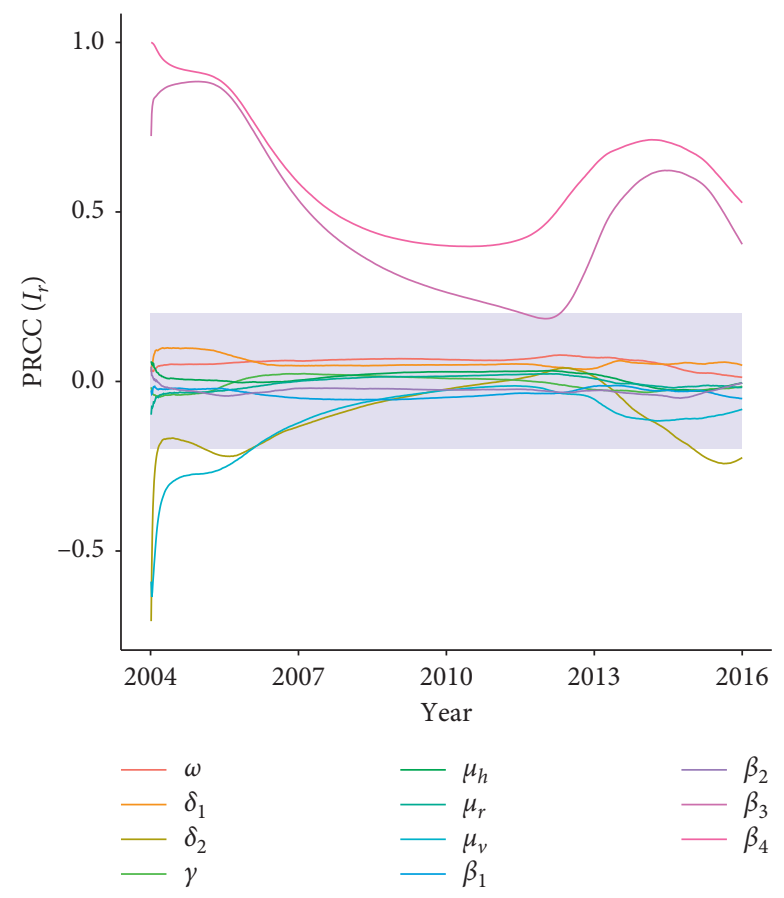

(c)

FIGURE 6: Sensitivity analysis: PRCC of the eleven parameters for (a) the number of infected humans, (b) the number of infected sandflies, and (c) the number of infected dogs.

Finally, in order to find better control strategies for VL transmission, we focus on changing the values of the parameters $\beta_{3}$ and $\beta_{4}$ to confirm the influence on the basic reproduction number $R_{0}$ (see Figure 8).

It can be seen from the figure that the parameters $\beta_{3}$ and $\beta_{4}$ have a strong influence on $R_{0}$, and the value of the basic reproduction number $R_{0}$ increased with the increases of $\beta_{3}$ and $\beta_{4}$; when $\beta_{3}$ is less than $6.854878 e-06$ or $\beta_{4}$ is less than $2.39872 e-06, R_{0}<1$, and the disease can be eliminated. This suggests that reducing the proportion rate between sandflies and dogs can effectively control the prevalence of VL. 


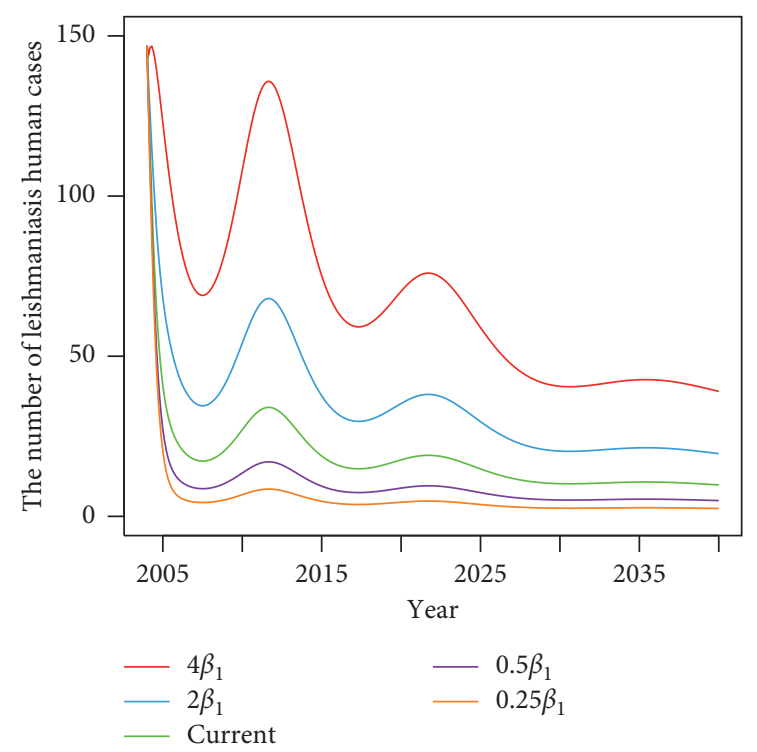

(a)

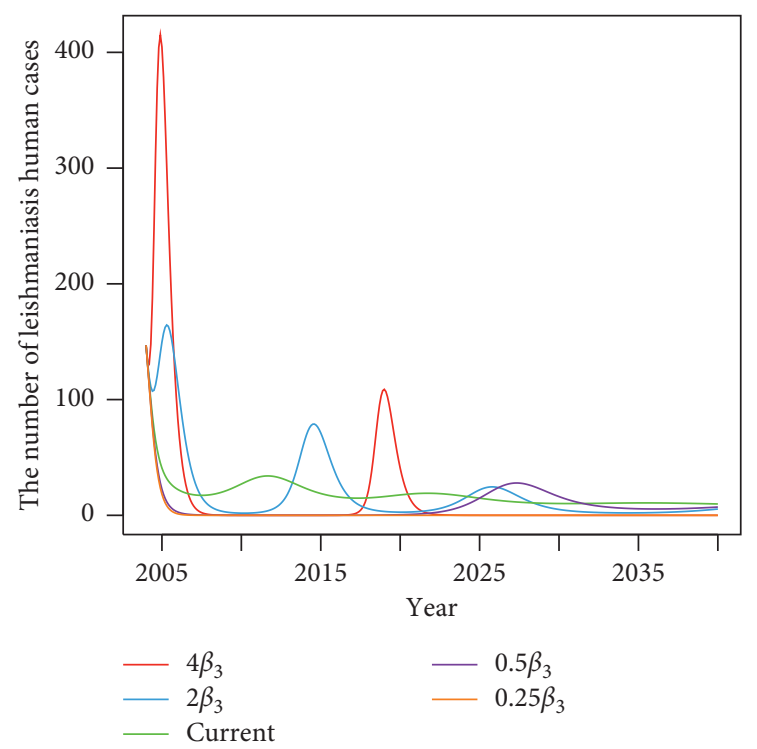

(b)

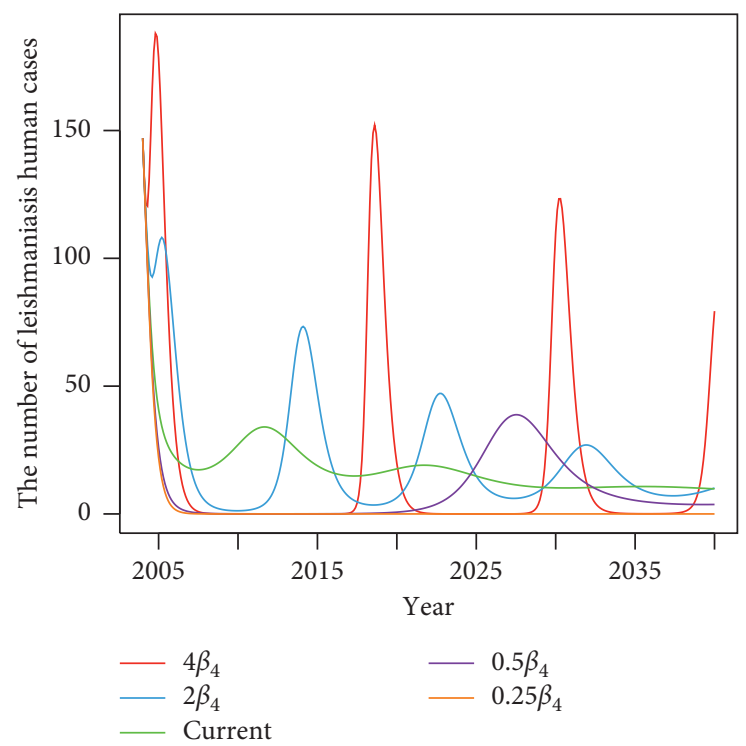

(c)

Figure 7: The influence of different values of parameters on the number of diseases of VL. (a) Versus $\beta_{1}$. (b) Versus $\beta_{3}$. (c) Versus $\beta_{4}$.

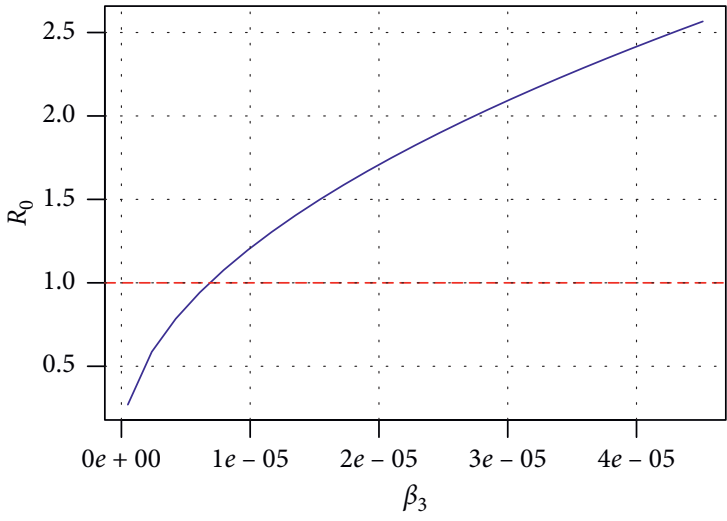

(a)

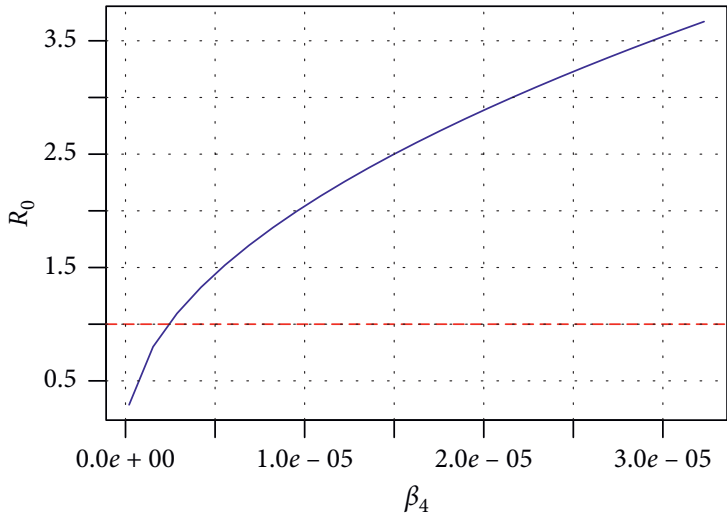

(b)

Figure 8: The influence of parameters on $R_{0}$ (red horizontal line is contour of $R_{0}=1$ ). (a) Versus $\beta_{3}$. (b) Versus $\beta_{4}$. 
Through the above sensitivity analysis results, we find that the main cause of the outbreak of VL is the mutual contact infection between dogs and sandflies. Therefore, we can get some effective strategies to reduce the prevalence of VL: the effective way is to reduce the contact between dogs and sandflies, and thus we can give the dog a collar with impregnated insecticide, increase the control of dog and reduce its active area, spray insecticides vigorously, etc.

\section{Conclusion and Discussion}

$\mathrm{VL}$ is a serious parasitic disease. It has been endangered for several decades in Xinjiang and has become a major parasitic disease affecting the local social and economic development. Kashgar is a high-risk area for the occurrence of VL. In order to reveal the spread of VL in Kashgar and predict the prevalence of VL, this paper proposes a dynamic model of VL propagation with logistic growth. The model describes the transmission of VL among humans, dogs, and sandflies.

We use model (1) to fit the cumulative data of VL in Kashgar. As it can be seen from the simulation results in Figure 2, our model is consistent with the actual data of the cumulative cases of VL in Kashgar. The results show that there are certain reliability and rationality to study the prevalence of VL in Kashgar using logistic growth of the VL model. Using model (1), the basic reproduction number is estimated to be 1.76 (95\% CI: 1.49-1.93) in the Kashgar Prefecture of Xinjiang. According to the threshold theory, it shows that VL will not disappear in the Kashgar area in a short time, and it may gradually become an endemic disease. According to the predictions for the next decade (see Figure 4), the cumulative incidence of VL in Kashgar is growing slowly, which means that the number of cases will gradually decrease over the next decade.

By selecting the sensitivity analysis of the parameters of interest for the basic reproduction number $R_{0}$ and the number of leishmaniasis human cases (see Figures 5 and 6), we can find that the most important factors affecting the basic reproduction number $R_{0}$ are $\beta_{3}$ and $\beta_{4}$, indicating that the infection between sandfly and $\operatorname{dog}$ has the greatest impact on $R_{0}$, and the most sensitive parameters affecting the number of leishmaniasis human cases are $\beta_{1}, \beta_{3}$, and $\beta_{4}$, which explains that the infection among the infected person, the dog, and the sandfly has the strongest impact on the number of diseases of VL. From Figure 7, we find that reducing the values of $\beta_{1}, \beta_{3}$, and $\beta_{4}$ can effectively reduce the value of the basic reproduction number $R_{0}$ and the number of leishmaniasis human cases. When the values of $\beta_{3}$ and $\beta_{4}$ drop to $6.854878 e-06$ and $2.39872 e-06$, respectively, the value of the basic reproduction number $R_{0}$ will decrease to 1, and VL will not be epidemic but gradually disappear in Kashgar. Therefore, in order to control the spread of the disease, effective strategies should be taken to prevent and control leishmaniasis in Kashgar; we can decrease the incidence of leishmaniasis in humans by reducing the contact between sandflies and dogs. Without considering costs, we can vector controls (e.g., environmental clean-up and insecticide sprayed around buildings) and dog controls (e.g., insecticide releasing dog collars and dog vaccinations).
In order to reduce the bite of the sandflies, we can use insecticidal bed nets. Meanwhile, we should conduct public education on the dangers and prevention of leishmaniasis for people. In short, the model we have established now can reflect the dynamics of VL in Kashgar Prefecture.

\section{Data Availability}

The data will be available upon request.

\section{Conflicts of Interest}

The authors declare that there are no conflicts of interest regarding the publication of this paper.

\section{Authors' Contributions}

Yateng Song and Tailei Zhang contributed equally.

\section{Acknowledgments}

This research was supported by the National Natural Science Foundation of China (grant no. 11961071).

\section{References}

[1] K. S. Rock, R. J. Quinnell, G. F. Medley, and O. Courtenay, "Chapter two-progress in the mathematical modelling of visceral leishmaniasis," Advances in Parasitology, vol. 94, pp. 49-131, 2016.

[2] A. Mubayi, C. Castillo-Chavez, G. Chowell et al., "Transmission dynamics and underreporting of Kala-azar in the Indian state of Bihar," Journal of Theoretical Biology, vol. 262, no. 1, pp. 177-185, 2010.

[3] C. J. Zheng, C. Z. Xue, W. P. Wu et al., "Analysis of epidemiological characteristics of black fever reported cases in China from 2005 to 2015," Chinese Journal of Epidemiology, vol. 38, no. 4, pp. 431-434, 2017.

[4] Kaisaier, C. Z. Zhu, W. P. Wu et al., "Reflections on prevention and control of VL in Kashgar, Xinjiang," Chinese Journal of Disease Control and Prevention, vol. 23, no. 5, pp. 23-24, 2008.

[5] L. Y. Wang, W. P. Wu, Y. Y. Guan et al., "Epidemiological characteristics of patients with high fever epidemic area in Kashgar, Xinjiang," Chinese Journal of Pathogenic Biology, vol. 8, no. 6, pp. 539-540, 2013.

[6] Z. Muhammad and R. Ali, "Zoonotic visceral Leishmania: modeling and control," Journal of Applied \& Computational Mathematics, vol. 4, no. 4, 2015.

[7] I. M. Elmojtaba, J. Y. T. Mugisha, and M. H. A. Hashim, "Mathematical analysis of the dynamics of visceral leishmaniasis in the Sudan," Applied Mathematics and Computation, vol. 217, no. 6, pp. 2567-2578, 2010.

[8] M. Zamir, R. Sultana, R. Ali, W. Panhwar, and S. Kumar, "Study on the threshold conditions for infection of visceral leishmaniasis," Sindh University Research Journal (Science Series), vol. 47, no. 3, pp. 619-622, 2015.

[9] K. Bi, Y. Chen, S. Zhaop, Y. Kuang, and C. H. John Wu, "Current visceral leishmaniasis research: a research review to inspire future study," BioMed Research International, vol. 2018, Article ID 9872095, 13 pages, 2018.

[10] Xinjiang Daily, http://www.xjdaily.com/. 
[11] C. Dye, "The logic of visceral leishmaniasis control," The American Journal of Tropical Medicine and Hygiene, vol. 55, no. 2, pp. 125-130, 1996.

[12] M. I. ELmojtaba, "Mathematical model for the dynamics of visceral leishmaniasis-malaria co-infection," Mathematical Methods in the Applied Sciences, vol. 39, no. 16, pp. 4334-4353, 2016.

[13] S. Zhao, Y. Kuang, C. H. Wu et al., "Zoonotic visceral leishmaniasis transmission: modeling, backward bifurcation, and optimal control," Journal of Mathematical Biology, vol. 73, no. 6-7, pp. 1525-1560, 2016.

[14] A. D. P. Sevá, M. Martcheva, T. Necibe et al., "Efficacies of prevention and control measures applied during an outbreak in southwest Madrid, Spain," PLoS One, vol. 12, no. 10, 2017.

[15] O. Diekmann, J. A. P. Heesterbeek, and J. A. J. Metz, "On the definition and the computation of the basic reproduction ratio $R_{0}$, in models for infectious diseases in heterogeneous populations," Journal of Mathematical Biology, vol. 28, no. 4, pp. 365-382, 1990.

[16] P. van den Driessche and J. Watmough, "Reproduction numbers and sub-threshold endemic equilibria for compartmental models of disease transmission," Mathematical Biosciences, vol. 180, no. 1-2, pp. 29-48, 2002.

[17] H. R. Thieme, "Persistence under relaxed point-dissipativity (with application to an endemic model)," SIAM Journal on Mathematical Analysis, vol. 24, no. 2, pp. 407-435, 1993.

[18] P. D. Leenheer and H. Smith, "Virus dynamics: a global analysis," SIAM Journal on Applied Mathematics, vol. 63, no. 4, pp. 1313-1327, 2003.

[19] H. Y. Hu, "A simple method for solving the accurate value of intra-phase growth rate in population," Anhui Agricultural Science Bulletin, vol. 16, no. 3, pp. 173-174, 2010.

[20] Statistics Bureau of Xinjiang Uygur Autonomous Region, http://www.kashi.gov.cn/Category_1008/Index.aspx.

[21] G. Wang, J. Wang, J. S. Li et al., "Further study on the ecology of sandfly in the Kashgar prefecture of Xinjiang," Chinese Journal of Disease Control and Prevention, vol. 7, no. 1, pp. 72-76, 1992.

[22] L. Guan, J. J. Chai, and X. P. Zuo, "Advances in biology of Xinjiang sandfly," Chinese Journal of Disease Control and Prevention, vol. 14, no. 4, pp. 87-91, 1999.

[23] Z. B. Zhou, D. G. Gu, L. R. Guan et al., "Changes in the composition ratio of main species of Kashgar prefecture in Xinjiang and its relationship with visceral leishmaniasis," International Journal of Medical Parasitic Diseases, vol. 42, no. 2, 2015.

[24] A. A. Pereira Filho, M. da Conceição Abreu Bandeira, R. S. Fonteles et al., "An ecological study of sand flies (diptera: psychodidae) in the vicinity of Lençóis Maranhenses National park, Maranhão, Brazil,” Parasites \&Vectors, vol. 8, no. 1, pp. 8-442, 2015.

[25] China News Network, http://www.sohu.com/a/122899918_ 123753.

[26] S. Marino, I. B. Hogue, C. J. Ray, and D. E. Kirschner, “A methodology for performing global uncertainty and sensitivity analysis in systems biology," Journal of Theoretical Biology, vol. 254, no. 1, pp. 178-196, 2008. 